\title{
Paper
}

\section{A P2P-based Communication Framework for Geo-Location Oriented Networks}

\author{
Takumi Miyoshi $^{1}$, Yusuke Shimomura ${ }^{2}$, and Olivier Fourmaux ${ }^{3}$ \\ ${ }^{1}$ College of Systems Engineering and Science, Shibaura Institute of Technology, Saitama, Japan \\ ${ }^{2}$ Graduate School of Engineering and Science, Shibaura Institute of Technology, Saitama, Japan \\ ${ }^{3}$ Laboratoire d'Informatique de Paris 6, Sorbonne Université, Paris, France
}

\begin{abstract}
This paper proposes a novel peer-to-peer communication framework to implement geographical location oriented networks, called G-LocON. Location-based services have been gaining in popularity, as proven by ridesharing and mobile games. Although these services have to construct geolocation oriented networks based on their users' geographical locations, they completely rely on client/server models to communicate with neighboring terminals. G-LocON provides geolocation oriented device-to-device communication only with the current wireless technologies, such as LTE and Wi-Fi, cooperating with the global positioning system and peer-to-peer overlay networking. G-LocON will serve as a type of a mobile ad-hoc network in which devices located within the focusing area are capable of communicating directly. We developed a primitive Android application to implement the GLocON framework. Evaluation of the solution's performance has verified the usefulness of the proposed system that offers an admissive transmission delay. Moreover, to confirm the application-related potential of the G-LocON framework, we also show a practical map software in which all neighboring mobile devices present in the focusing area are displayed.
\end{abstract}

Keywords-geolocation oriented network, location-based service, overlay, peer-to-peer.

\section{Introduction}

In the past decade, smartphones have been expanding the range of their functionalities and have been gaining in popularity - not only mobile phones, but also as portable computing devices. Some reports forecast that the number of smartphone users in the world will reach 2.7 billion and will exceed $50 \%$ of all mobile users in 2019 [1], [2]. One of the remarkable smartphone functions is the global positioning system (GPS). GPS is capable of pinpointing the geographical location of users based on signals received from four or more GPS satellites, without any data transmission from GPS receivers. Ohmae defines a generation-based evolution model for the location information (LI) business [3]. The history of LI started in the 1990s with digital map services, including car navigation and digital map services on personal computers. In this generation, LI was used personally and privately to display the user's location on the map. In the 2000s, the advent of social networking services (SNS) and smartphones has brought about the next generation solution - LI 2.0. Users commonly provide their own LI to smartphone applications, some of which send LI to servers, where it is registered, and may therefore enjoy geolocation-based services (LBS). Ohmae estimates that we are now opening the door to the LI 3.0 era, in which LBS will be much improved and enhanced by Internet of Things (IoT) devices and sensors around us.

As far as geolocation-based applications are concerned, digital maps came first, route search and navigation still remain the areas in which the service is much more popular and useful. SNS applications, such as Facebook, Twitter, and Instagram, are also installed on most user smartphones. By tagging and sharing the users' locations, trajectories and data on where they are/were or what they intend to visit, the applications may easily find useful information, such as reputations and recommendations of shops and popular places. Ridesharing services, such as Uber and Grab, integrate LI and SNS. Digital map applications show the user's position, as well as the vehicles available around the user, and social networks establish trust and accountability between passengers and drivers [4], [5]. Furthermore, augmented reality (AR) has become, in recent years, a popular application that relies on the user's LI. Sekai Camera [6] has brought about a new world where, through the smartphone camera, we can see the messages and photos that are saved and linked to the real world by means of LI tags assigned by other users. Niantic [7] launched megahit games, Ingress and Pokemon Go, and is the world's leader in AR gaming.

Following in the footsteps of the LI services referred to above, inter-vehicular communication is one of the promising core technologies in intelligent transportation systems (ITS) [8], [9]. In general, vehicular-related communication forms a vehicular ad hoc network (VANET) among neighboring devices by utilizing radio propagation-based direct communication [10] and infrastructure-based communication [11]. VANET thus realizes a close-range direct or multi-hop communication among vehicles and other peripherals [12]. 
As described above, geolocation-oriented communications have good prospects for the future. However, there are some problems and challenges in LBS. Firstly, the current location-based applications are mainly realized as serverbased systems. User devices and their LI are registered on and maintained at the location servers, and each device has to connect to the servers to obtain information about objects around the user. The computation- and storagerelated load would be therefore concentrated on the servers. Secondly, although multi-hop communication has certainly been a promising technology for more than 20 years now, no one knows when it is due to become widespread and installed on consumer devices. Several research groups have attempted, in recent years, to evaluate VANET communications using IEEE 802.11p [13]-[15]. However, most conventional studies focus solely on fundamental characteristics of packet transmissions between vehicles, such as packet delivery ratio, transmission delay and jitter. Moreover, IEEE 802.11p devices have not yet become common in consumer products. The VANET system will work well after all vehicles have been equipped with wireless communications solutions, and it has to be borne in mind that people do not tend to replace their cars with new ones very frequently.

In this paper, we propose G-LocON - a novel communication framework that may be relied upon to establish geographical location oriented networks. G-LocON constructs a peer-to-peer (P2P) logical overlay network around each user. We implement a location tracker, which maintains LI of the user's devices and helps discover neighboring devices. Therefore, the proposed framework provides geolocation oriented device-to-device communication. Since the system relies solely only on such current technologies as LTE, Wi-Fi and GPS, the public may enjoy this service of the future on their smartphones without any delay.

The remainder of the paper is organized as follows. Section 2 briefly discusses previous works related to LBS and location-based P2P networks. In Section 3, we explain the G-LocON framework and describe the proposed protocols, as well as an implementation method. Section 4 shows a primitive application to realize the G-LocON framework and reports the results of its experimental evaluations in the actual environment, from the viewpoint of transmission delay. In Section 5, in order to confirm the potential of the G-LocON framework, we will demonstrate a practical Android application in which all neighboring mobile devices positioned within the focusing area are displayed on a peripheral street map. Conclusions and suggestions for future work are provided in Section 6.

\section{Related Work}

LBS is one of the recent and hot topics and it has succeeded in attracting much attention from numerous researchers [16]-[24]. Popular research objectives are mainly related to LBS frameworks [16]-[18] and LBS applications [19], [20], [23], [24]. In the former category, authors propose new network architectures or frameworks to provide location-based services. The latter category, on the other hand, develops new applications, such as mobile positioning or navigation systems.

Some articles utilize the P2P approach, which we also focus on in this paper, to construct logical overlay networks to discover neighbor peers [21]-[24]. Kaneko et al. propose a tree-based P2P overlay structure called LL-Net [21]. They assume a mixed environment where both fixed and mobile devices coexist, and try to form a three-layer tree structure on a traditional grid-cutting region model. LL-Net was evaluated for content retrieval by means of a computer simulation. Kovačević et al. also propose a hierarchical tree-based P2P overlay called Globase.KOM [22]. They focus on a world-wide spanning network, which is divided into non-overlapping rectangular zones. Simulation results show that Globase.KOM achieves full retrievability of area searches and a short response time. Wang et al. develop a $\mathrm{P} 2 \mathrm{P}$ mobile navigation system to guide visitors in a flora exposition with 3D renders [23], [24]. P2P technology is used for clustering neighboring users who have similar interests and for downloading data from them. They also validate faster transmission rates of the proposed system by computer simulation, assuming all devices are connected to $\mathrm{Wi}-\mathrm{Fi}$.

The conventional location-based P2P systems mentioned above still suffer from certain problems. Both LL-Net and Globase.KOM target wide-area content retrievals and then form hierarchical tree-based overlay networks. This approach is costly in terms of finding super peers and constructing hierarchical networks when the focus is placed on a small area around each user. Moreover, all systems mentioned above seem not to consider real-time communications between users. In addition, they can hardly be implemented as-is in the current mobile network environment due to the private addressing system they rely on. We believe that a hybrid P2P system in which some servers exist for maintaining peers helps identify neighbor peers in a quicker manner. For reference, a hybrid P2P system is a certain kind of an implementation form and is very commonly used in popular P2P file sharing and video streaming applications, such as BitTorrent, PPTV and PPStream.

\section{G-LocON Communication Framework}

\subsection{Overview of G-LocON}

This paper proposes a P2P-based communication framework to realize a geographical location oriented network, and is called G-LocON. An outline image of the proposed system is shown in Fig. 1. Here, mobile devices, i.e. peers, are assumed to connect to various networks: they connect to cellular networks, Wi-Fi and so on. G-LocON provides a hybrid P2P network by implementing a location tracking server called L-tracker. L-tracker maintains the peers' information that consists of peer identifiers and lo- 
cations, and helps each peer discover the neighbor peers around it.

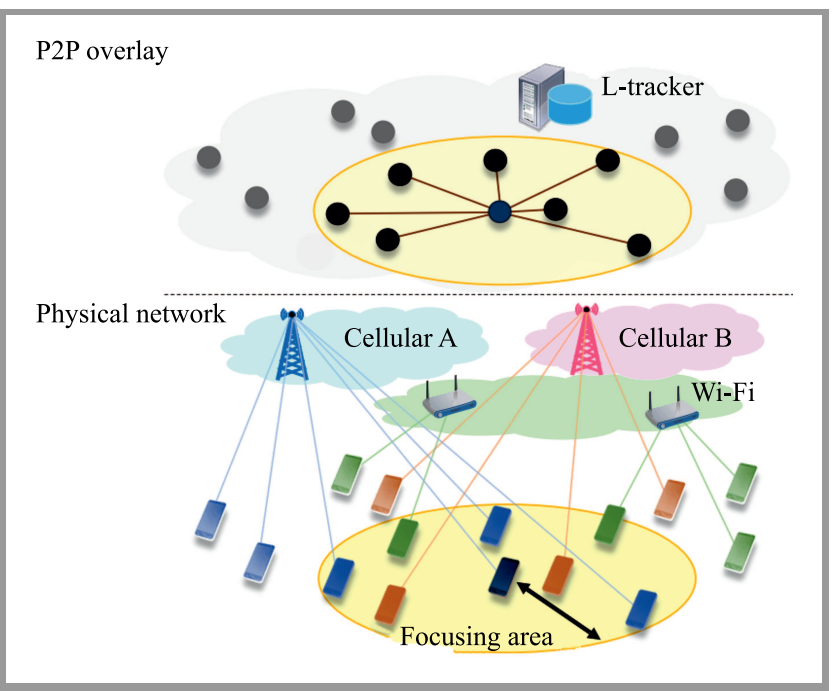

Fig. 1. G-LocON framework: forming a P2P overlay managed by L-tracker on interconnected physical networks.

G-LocON, by nature of a hybrid P2P system, may flexibly manage such unstable logical overlay networks formed by the dynamically moving focusing areas. Furthermore, each user is free to decide the size of its focusing area, since the range within which communication with neighbor peers may be established is unlimited. All peers may connect to each other through the Internet, independently of user density. This is a major advantage over ad hoc networks in which mobile devices hardly form a network in an area with sparsely located users, unless they are located mutually within the range of their wireless communication systems.

When a new peer joins the proposed system, it sends, firstly, a join message with peer information and its LI to the L-tracker. The peer then requests neighbor peers information from the L-tracker, and finally establishes P2P connections to its neighbors. In the proposed system, every peer periodically registers its LI with the L-tracker by reusing the join message, obtains renewed information about neighbor peers and updates the connections to its neighbors. After establishment of P2P connections, the peer directly communicates with its neighbors without relaying data through any servers. This is a typical behavior of hybrid P2P systems.

\subsection{How to Form the G-LocON Overlay}

To establish a direct connection between two mobile devices, a peer needs to know the translated IP address and port number of the destination peer. Such information is called address bindings and is automatically allocated when a packet crosses a NAT gateway. In this paper, a STUN server [25] is deployed to resolve the bindings information. Moreover, peers also have to exchange metadata to coordinate communication. Therefore, we introduce a signaling server to share the information about peers and to coordinate connections between them.

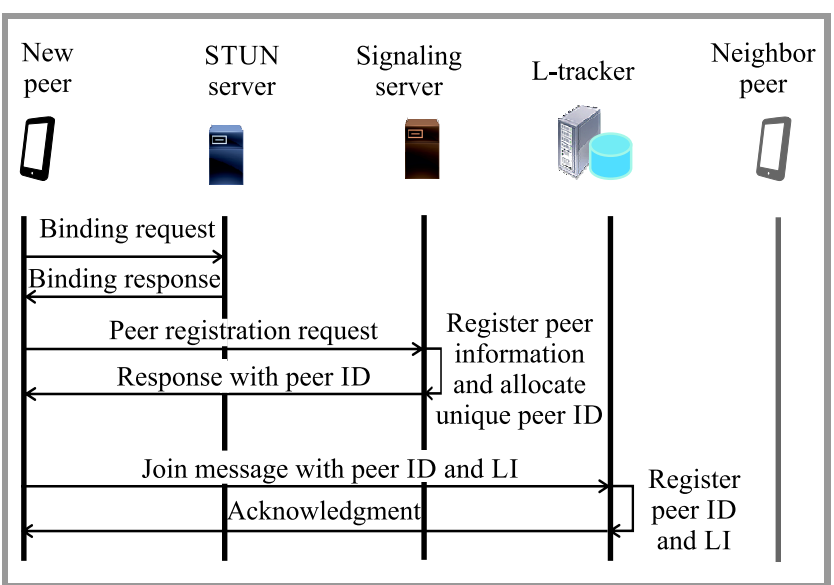

Fig. 2. Process of joining G-LocON.

The process of joining G-LocON. Figure 2 shows the communication process that takes place when a new peer joins the G-LocON overlay network. The sequence of process is described as follows:

- Firstly, the new peer sends a binding request to the STUN server. The server provides a binding response that consists of the IP address and port number, as observed from the server's perspective;

- The new peer then sends a registration request to the signaling server. The request message contains the peer's IP address and port number resolved by the STUN server. The server allocates a unique identifier to the peer (peer ID) and registers these three types data in the database. The server also sends back the peer ID to the peer as a response;

- The peer sends a join message to the L-tracker. The message includes the peer ID and LI that is obtained by the GPS module. The L-tracker then registers them in the database and replies with an acknowledgment. This step will be periodically repeated for updating LI at intervals of $T_{\text {int }}$.

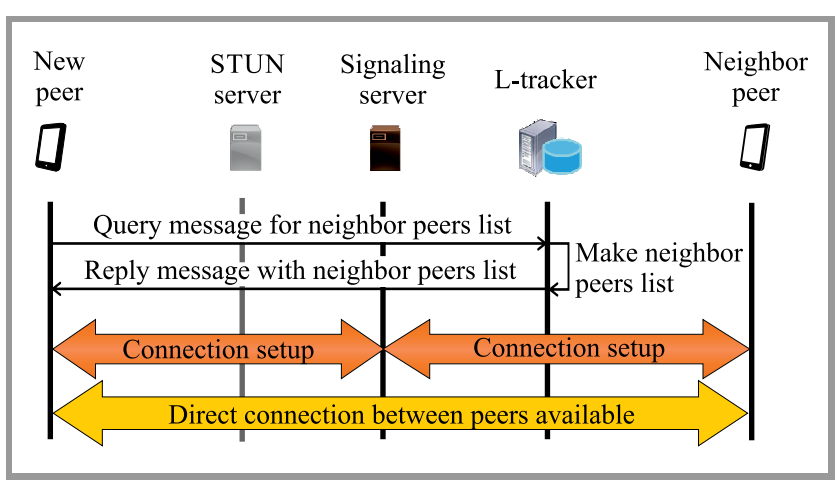

Fig. 3. P2P connection process. 
Establishment of P2P connection between peers. After the joining process has been completed, the peer becomes ready to establish $\mathrm{P} 2 \mathrm{P}$ connections. Figure 3 shows the communication process. When a peer sends a neighbor search query to the L-tracker, the focusing area to be searched has to be clearly indicated. In this paper, we determine that each focusing area is a circular form that is defined by a center and a radius. The center point may be the peer's location, and the radius represents the size of the focusing area. The sequence of the $\mathrm{P} 2 \mathrm{P}$ connection process is described as follows:

- The peer sends a query message to the L-tracker to discover neighbor peers. The message includes the focusing area information, such as the peer's LI and a radius. The L-tracker makes a list of neighbor peers found in the focusing area and then sends it back to the peer, as a reply message. The peer maintains a list which consists of peer IDs only;

- Based on the list of neighbor peers, the peer sets up $\mathrm{P} 2 \mathrm{P}$ connections with its neighbor peers, relying on the assistance of the signaling server. This process enables the peer to obtain the IP addresses and port numbers of each neighbor peer. The server sends another signaling message to each neighbor peer to inform it of the connection request from the new peer, as well as to provide its IP address and port number. Herewith, the two peers may establish the P2P connection and may subsequently communicate with each other any time while the connection remains alive. Each peer maintains the list of P2P connections;

- The peer closes its $\mathrm{P} 2 \mathrm{P}$ connections if the neighbor peers that have already been connected to disappear from the list of neighbor peers.

If there is a particular type of NAT between two peers, the connection cannot be established by the above sequence. In this case, the peers try to connect through a TURN server [26]. Moreover, the P2P connection process is executed after each periodic repetition join messages sent to L-tracker. The interval time is thus $T_{\text {int }}$.

The process of leaving G-LocON. Figure 4 shows the communication process when a peer leaves the G-LocON overlay network. The sequence of the leaving process is described as:

- The peer first closes all the P2P connections with its neighbor peers, by sending connection closing messages;

- The peer sends a leave message to the L-tracker. The L-tracker then deletes relevant information from the database;

- The peer sends a peer unregistration request to the signaling server. The server then deletes relevant information from the database.

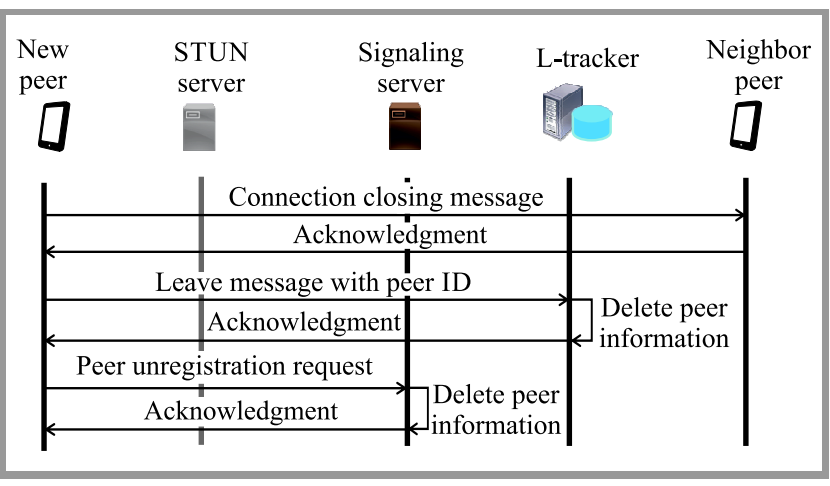

Fig. 4. The process of leaving G-LocON.

Avoidance of multiple P2P connections. If two peers attempt, virtually simultaneously, to initiate $\mathrm{P} 2 \mathrm{P}$ connections with each other, multiple connections may be established occasionally. We therefore prepare a process to avoid such multiple connections. When a peer detects a new P2P connection with a neighbor peer, the peer checks if another connection with the same peer ID exists in the list of P2P connections. If it does, the new connection is immediately closed. Otherwise, the list is updated by adding information about the new connection.

\subsection{Implementation}

We implemented the G-LocON framework to establish geolocation-based P2P overlay networks. The L-tracker was developed on NIFCLOUD, a public cloud computing service provided by Fujitsu Cloud Technologies [27]. We also used SkyWay API, a WebRTC platform provided by NTT Communications [28]. SkyWay also offers the STUN, TURN, and signaling services. The mobile software was developed with the use of Android Studio with NIFCLOUD and SkyWay APIs. Asus ZenFone 3 / 3 Laser / 2 Laser, LG Nexus 5 / 5X, Samsung Galaxy S6, and Sony Xperia ZL2 were used as Android-based smartphones.

In the application developed, each peer obtains its LI from the GPS module every second, as well as registers and updates LI to the L-tracker every five seconds: $T_{\text {int }}=5 \mathrm{~s}$. After establishing P2P connections with neighbor peers, each peer tries to share and send LI to its neighbors as soon as LI has been updated, i.e., every second. Each peer will maintain LI received from its neighbor peers.

\section{Performance Evaluation}

\subsection{Experimental Settings}

To evaluate the performance of G-LocON, we performed experiments to form a P2P overlay network comprising several Android smartphones operating in the real-world environment. In the experiments, we focused primarily on communication and processing delays. Here, $t_{l}, t_{s}$, and $t_{r}$ denote the times when a peer obtains its LI from the GPS module, when the peer starts sending LI to each neighbor peer, and when the neighbor peer receives LI, respectively. 
These times were recorded in each smartphone by relying on the GPS signal and its internal clock that had been adjusted beforehand by the network time protocol (NTP). $T_{\text {send }}=t_{r}-t_{s}$ represents the transmission delay required for a peer to send its LI to each neighbor peer. Meanwhile, $T_{\text {total }}=t_{r}-t_{l}$ represents the total delay time from when a peer obtains its LI to when LI arrives at each neighbor. In this paper, we evaluate $T_{\text {send }}$ and $T_{\text {total }}$.

Two situations have been considered, namely a static and a dynamic scenario. In the static case, two to nine smartphones were placed in the laboratory, next to a window, to enable GPS signals to be received without any problems, and were connected to our university's Wi-Fi network. The G-LocON overlay established by those devices formed a full mesh topology since all peers were within the focusing areas of the other peers. Therefore, the smartphones mutually regarded each other as their neighbor peers. In one experiment set, each peer sends its LI to the remaining peers 300 times. Three sets have been performed and the mean values of $T_{\text {send }}$ and $T_{\text {total }}$ were calculated. In the dynamic case, two to five persons randomly moved with their smartphones, walking with a specified open-air area. The devices connected to LTE cellular networks provided by well-known Japanese mobile service providers, au (KDDI) and IIJ mobile (MVNO on NTT DOCOMO). The form of the G-LocON overlay was not always of the full-mesh variety, since some peers occasionally moved out of the focusing areas of other peers. We performed only one experimental set and calculated the mean values of $T_{\text {send }}$ and $T_{\text {total }}$.

\subsection{Results}

Figures 5 and 6 present the average transmission delay $T_{\text {send }}$ and the average total delay $T_{\text {total }}$ in the static case, respectively. In a similar fashion, Figs. 7 and 8 show the results in the dynamic case. In each figure, we indicate three types of delay values: the overall average delay (blue line and circles), the average delay when peers obtain the updated list of neighbor peers from the L-tracker (red line and squares), and the average delay when the updated list is not obtained (green line and triangles).

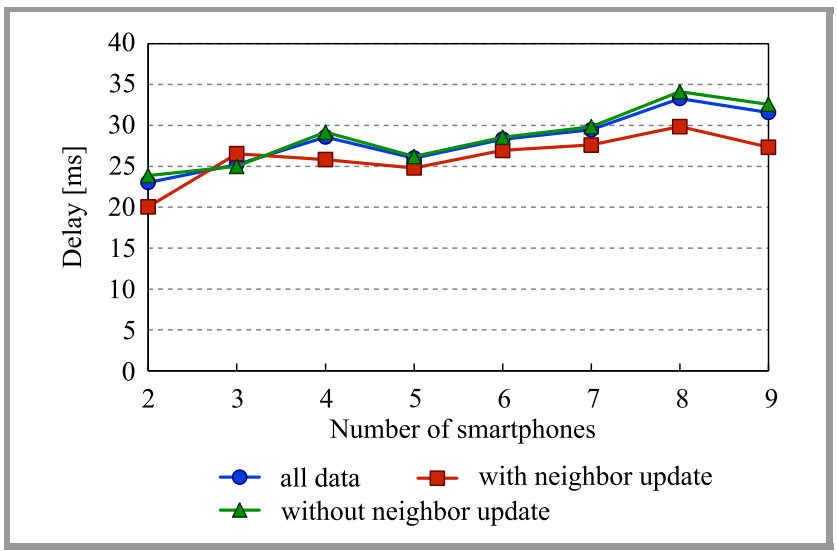

Fig. 5. Average transmission delay $T_{\text {send }}$ (static case).

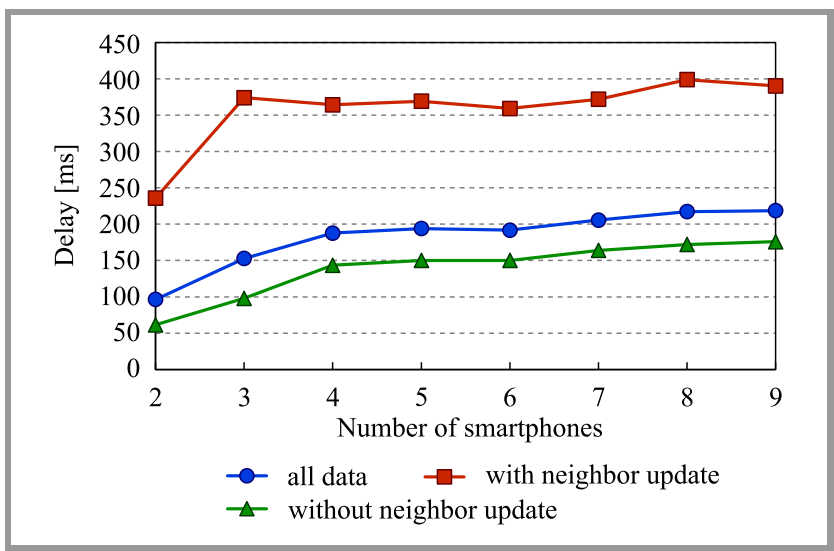

Fig. 6. Average total delay $T_{\text {total }}$ (static case).

As shown in Fig. 5, the average transmission delay $T_{\text {send }}$ becomes gradually longer with an increase in the number of devices. This is because both transmission and processing load of each peer become large as the number of neighbor peers increases. On the other hand, the delay when peers receive the updated list of neighbor peers (red line) is lower than the delay when the list is not obtained (green line). For updating the neighbor peers, each peer connects to the L-tracker at intervals of $T_{\mathrm{int}}$. Since this process is executed sequentially in our current implementation, the peer does not communicate with its neighbor peers; and consequently the processing load will be reduced. From Fig. 6, it is observed that the average total delay $T_{\text {total }}$ becomes much larger than the transmission delay $T_{\text {send }}$. In particular, the red line is roughly twice as high as the blue line. The total delay represented by the red line includes the processes required to obtain the list of neighbor peers from the L-tracker and to establish $\mathrm{P} 2 \mathrm{P}$ connections with the neighbors. These processes take 170 to $270 \mathrm{~ms}$, with the said values obtained as differences between red and green lines. Based on the results presented above, we are of the opinion that the performance of G-LocON depends strongly on communication with L-tracker and on the establishment of $\mathrm{P} 2 \mathrm{P}$ connections.

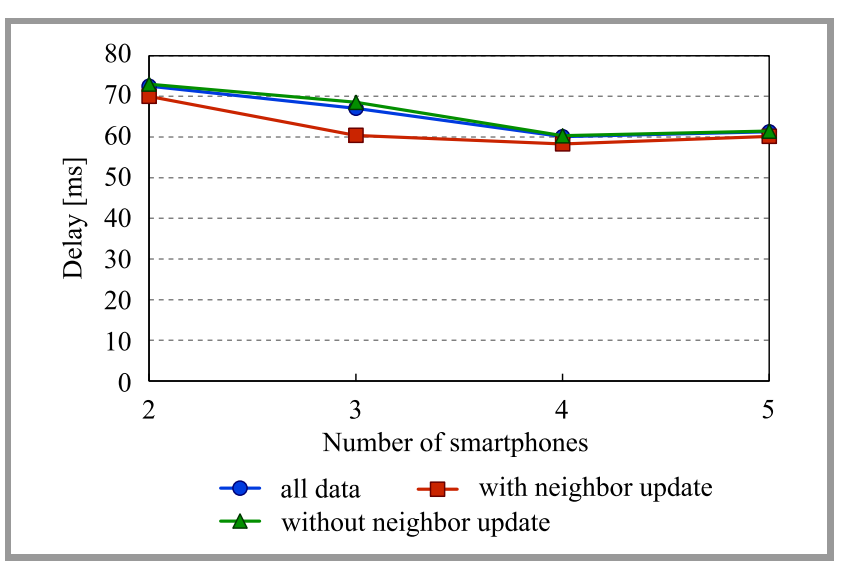

Fig. 7. Average transmission delay $T_{\text {send }}$ (dynamic case).

Next, we turn to the dynamic case. It may be seen in Fig. 7 that the average transmission delay $T_{\text {send }}$ reaches more 
than twice the value observed in the static case. This is because a longer network delay was added, since all smartphones were connected to LTE networks. However, the value does not deteriorate along with an increase in the number of devices. This phenomenon may be potentially explained by the fact that the cellular network connectivity varied by the hour, and the bandwidth available changed accordingly. To be honest, however, further experiments and thorough measurements are needed with more smartphone devices in the dynamic case.

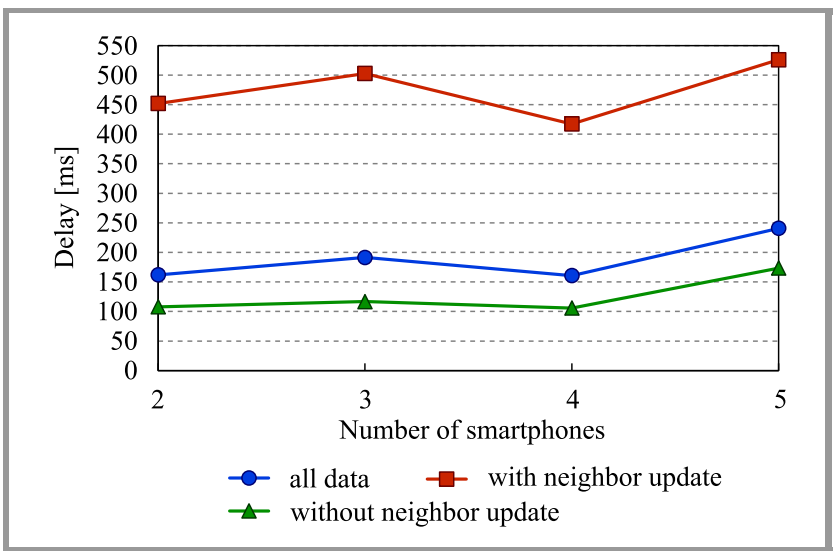

Fig. 8. Average total delay $T_{\text {total }}$ (dynamic case).

As shown in Fig. 8, the average total delay $T_{\text {total }}$ has similar characteristics to those observed in the static scenario: The total delay when the neighbor peers list was updated is much longer than in the case without the update. This also makes it clear that communication with the L-tracker and the establishment of $\mathrm{P} 2 \mathrm{P}$ connections take a relatively long time compared with direct $\mathrm{P} 2 \mathrm{P}$ communications between the peers.

As far as this aspect is concerned, we carefully examined the log data related to a situation in which three smartphone devices formed the G-LocON overlay in the dynamic case. We focused our attention on how long it takes for a peer to completely establish a $\mathrm{P} 2 \mathrm{P}$ connection after it receives the list of its neighbor peers. From the observation, we found that cases existed in which the time required equaled several seconds. The time scale of this waiting time cannot be neglected when the G-LocON framework is used for real-world applications, such as inter-vehicular communications. Since the delay time will probably increase with the number of peers, implementation of the protocol to communicate with the L-tracker and the processing methods relied upon to establish P2P connections should be improved considerably.

\section{Practical Application of G-LocON Framework}

\subsection{Application Design}

To confirm the potential of the proposed G-LocON framework, we developed practical software for Android smart- phones. We believe that G-LocON may be applied in intervehicular or in vehicle-to-pedestrian communications. In the developed application, assuming that both drivers and pedestrians use their own smartphones, the locations and moving speeds of neighbor peers present in the focusing area are shown on a peripheral street map.

The principal operation sequence of the developed software is based on the processes explained in Subsection 3.2, as described below:

1. A peer first joins the G-LocON overlay network. The joining process is repeated every five seconds: $T_{\text {int }}=5 \mathrm{~s}$;

2. The peer sends a query message to the L-tracker to discover neighbor peers, and then obtains the list of neighbor peers in the circular focusing area whose radius equals $50 \mathrm{~m}$;

3. The peer sets up $\mathrm{P} 2 \mathrm{P}$ connections with its neighbors. They exchange their actual locations and moving velocities, relying on P2P communication, every second. The moving velocity of peer $i$, denoted by $\boldsymbol{v}_{i}(t)$, can be calculated as the difference between current and previous position vectors,

$$
\begin{aligned}
\boldsymbol{v}_{i}(t)=\boldsymbol{P}_{i}(t)-\boldsymbol{P}_{i}(t-\Delta t) \\
=\left[x_{i}(t)-x_{i}(t-\Delta t), y_{i}(t)-y_{i}(t-\Delta t)\right],
\end{aligned}
$$

where $\boldsymbol{P}_{i}(t)=\left(x_{i}(t), y_{i}(t)\right)$ represents position of peer $i$ 's at time $t$. $\Delta t$ represents the interval in which LI is obtained the GPS module, and $\Delta t=1 \mathrm{~s}$.

4. The peer's current location and circular focusing area are displayed on the peripheral map by relying on the Google map API. All neighbor peers are plotted concurrently as pins on the map. If the moving speed of a neighbor peer is $v_{\text {th }}$ faster than own speed, the color of pin becomes red to alert the user about a moving vehicle approaching in the vicinity. The other neighbors are shown as green pins. In this implementation, $v_{\text {th }}$ is set to $10 \mathrm{~km} / \mathrm{h}$.

5. When the user taps a pin that represents a neighbor peer, a popup balloon (known as toast in Android) appears to show the following information: peer's name, location, and moving speed.

\subsection{Field Experiment}

To confirm the behavior of the application, we performed a small field experiment. The software developed was installed on seven Android smartphones. All devices connected to the Internet using LTE cellular networks or Wi-Fi tethering via other mobile phones' hotspots.

Figure 9 shows the experimental environment in a residential area. In the experiment, two vehicles and five pedestrians used the application simultaneously. They can recognize one another as a neighbor peer if they enter the focusing areas of other peers. Two vehicles are approaching the intersection from outside of the pedestrians' focusing 


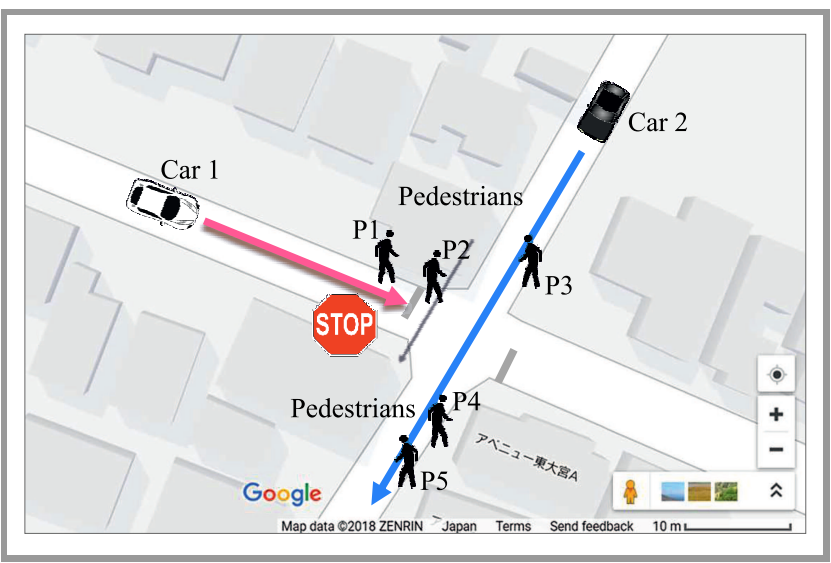

Fig. 9. Experimental environment for practical applications.

areas. Car 1 has to stop at a stop sign and wait for car 2 to by. Both vehicles obviously have to be careful and mind the pedestrians.

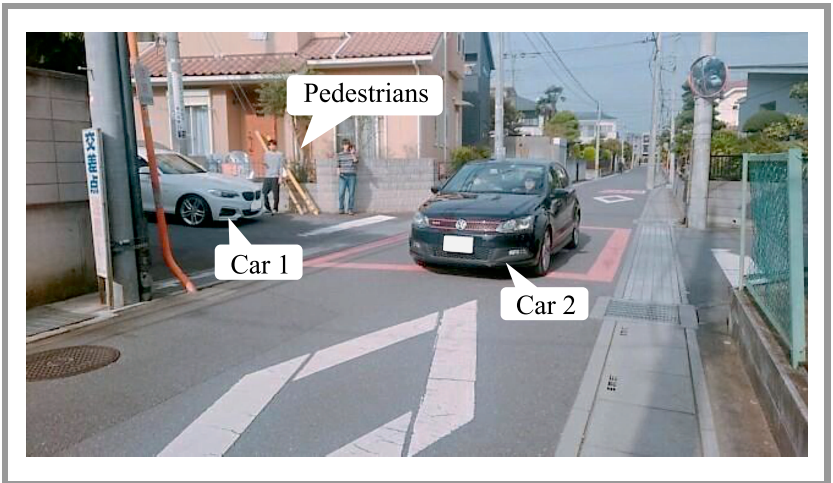

Fig. 10. A scene of the experiment when car 2 is passing an intersection: pedestrian P4's view.

The field experiment is shown in Fig. 10, with the image taken from the location of pedestrian P4. In this figure, car 2 is running through the intersection in front of car 1 which stops at the stop sign. The screenshots of Android smartphones are shown in Fig. 11. Figure 11a is P4's screenshot, and it only displays four other pedestrians because the vehicles have not entered the focusing area yet. On the contrary, Fig. 11b shows that two vehicles are entering the focusing area and are plotted by red pins. The users are therefore aware of the fact that moving vehicles are approaching. Figure 11c shows that the driver of car 1 is aware of car 2 running from left to right, as well as of some pedestrians. This application will certainly help drivers recognize other vehicles and pedestrians even at intersections with poor visibility. Finally, in Fig. 11d, a popup balloon appears to show information about car 1 when the user taps the pin of car 1. The peer's name, location and moving speed are obtained through $\mathrm{P} 2 \mathrm{P}$ communication between car 1 and car 2 .

Although the application developed was rudimentary in nature, the screenshots shown above clearly suggest the potential of the G-LocON framework. Namely, the proposed P2P mobile network overlay successfully conducts geolo-

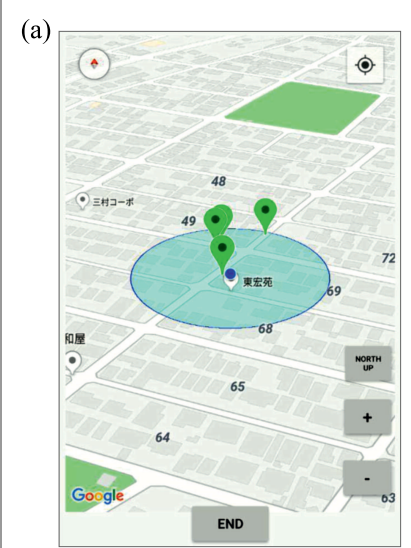

P4's view: no vehicle has existed yet in the focusing area.

(c)

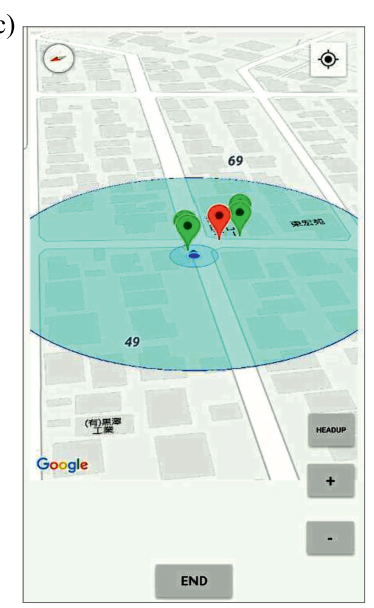

Car 1's view: car 2 is passing by in front of car 1 .

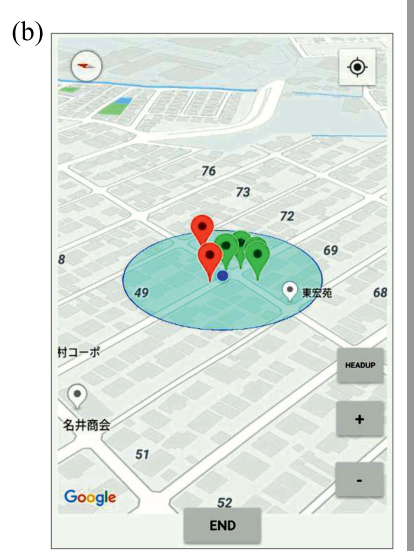

P1's view: two vehicles are entering the focusing area.

(d)

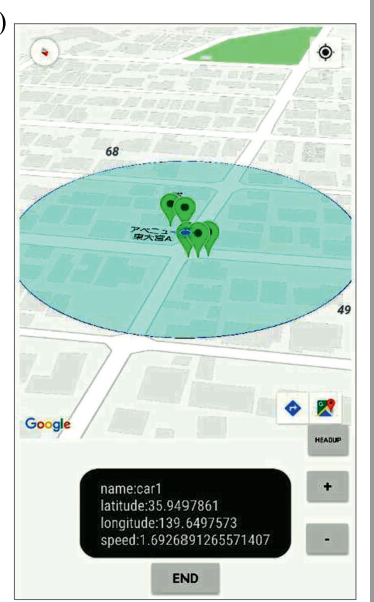

Car 2's view: the information of car 1 is popping up.

Fig. 11. Smartphone screenshots.

cation-based device-to-device communication relying only on current technologies. We are confident that the G-LocON framework will soon bring us to future LI services, such as inter-vehicular or vehicle-to-pedestrian communications.

\section{Conclusions}

In this paper, we proposed a G-LocON framework to establish a P2P-based geolocation-oriented network. By using the proposed scheme, each mobile device can easily discover its neighbors around and may then form a G-LocON overlay network. We firstly developed a primitive Android application to implement the G-LocON framework by utilizing public cloud computing and WebRTC platform services. The results obtained from the evaluation experiments can be summarized as follows:

- The average transmission delay between peers, represented as $T_{\text {send }}$, is of the order of tens of milliseconds, and gradually increases with the number of neighbor peers; 
- The average total delay from the moment a peer obtains its LI from the GPS module to the moment LI arrives at each neighbor, represented as $T_{\text {total }}$, is of the order of hundreds of milliseconds, and gradually increases with the number of neighbor peers;

- When peers obtain the updated list of neighbor peers from the L-tracker, the total delay becomes much longer than that without the update. Several seconds are occasionally needed to initiate a $\mathrm{P} 2 \mathrm{P}$ connection after the neighbor peers list arrives.

We also developed another practical Android application based on the G-LocON framework, which realizes P2Pbased direct neighbor communications to exchange information about peers between vehicles and pedestrians. Although the functionalities of the application are elementary, the small field experiment with vehicles and pedestrians has given an indication of the future potential of the G-LocON framework.

By analyzing the results of experiments described above, we have identified some problems concerning the G-LocON framework that require to be solved. Firstly, the protocol relied upon to communicate with the L-tracker and the processing methods establishing P2P connections should be much improved for larger-scale real-time applications. We will strive to introduce parallel processing in the communications between the L-tracker and neighbor peers. Secondly, public cloud services probably have their limits, such as the maximum number of connections, processing power and transmission speeds. We are now trying to implement our own L-tracker, which operates independently, without any cooperation with public cloud platforms. Finally, since the practical Android application developed in this paper was of the rudimentary nature, it was only capable of exchanging basic information between neighbors. We will strive to develop a more sophisticated application that may be used in a wide variety of environments.

In addition, we will evaluate the characteristics of the proposed system when user density changes.

\section{Acknowledgment}

This study was supported by JSPS Kakenhi Grant Number $17 \mathrm{~K} 06441$.

\section{References}

[1] Statistica, "Number of smartphone users worldwide from 2014 to 2020", 2018 [Online]. Available: https://www.statista.com/ statistics/330695/number-of-smartphone-users-worldwide

[2] Statistica, "Number of mobile phone users worldwide from 2013 to 2019”, 2017 [Online]. Available: https://www.statista.com/ statistics/274774/forecast-of-mobile-phone-users-worldwide

[3] K. Ohmae, "Business model in location information 3.0 era", Kenichi Omae's special lecture, biblion [Online]. Available: https://biblion.jp/articles/RTuqy [in Japanese]

[4] A. Amey, J. Attanucci, and R. Mishalani, "Real-time ridesharing the opportunities and challenges of utilizing mobile phone technology to improve rideshare services", Transportation Res. Record: J. of the Transport. Res. Board, vol. 217, no. 1, pp. 103-110, 2011 (doi: 10.3141/2217-13).
[5] S. Ma, Y. Zheng, and O. Wolfson, "Real-time city-scale taxi ridesharing”, IEEE Trans. Knowl. \& Data Eng., vol. 27, no. 7, pp. 1782-1795, 2015 (doi: 10.1109/TKDE.2014.2334313).

[6] Tonchidot, "Introduction of sekai camera", Youtube video, Nov. 2010 [Online]. Available: https://www.youtube.com/ watch?v=oxnKOQkWwF8

[7] Niantic, Inc., homepage [Online]. Available: https://www.nianticlabs.com

[8] H. Hartenstein and K. P. Laberteaux, "A tutorial survey on vehicular ad hoc networks", IEEE Commun. Mag., vol. 46, no. 6, pp. 164-171, 2008 (doi: 10.1109/MCOM.2008.4539481).

[9] B. T. Sharef, R. A. Alsaquor, and M. Ismail, "Vehicular communication ad hoc routing protocols: a survey", J. of Netw. \& Comp. Appl., vol. 40, pp. 363-396, 2014 (doi: 10.1016/j.jnca.2013.09.008).

[10] D. Jiang and L. Delgrossi, "IEEE 802.11p: towards an international standard for wireless access in vehicular environments", in Proc. IEEE Veh. Technol. Conf. VTC-Spring, Singapore, 2008 (doi: 10.1109/VETECS.2008.458).

[11] Qualcomm, "Creating a digital 6th sense with LTE direct", 2015 [Online]. Available: https://www.qualcomm.com/media/documents/ files/creating-a-digital-6th-sense-with-lte-direct.pdf

[12] L. Bariah, D. Shehada, E. Salahat, and C. Y. Yeun, "Recent advances in VANET security: a survey", in Proc. 82nd IEEE Veh. Technol. Conf. VTC2015- Fall, Boston, MA, USA, 2015 (doi: 10.1109/VTCFall.2015.7391111).

[13] F. A. Teixeira, V. F. e Silva, J. L. Leoni, D. F. Macedo, and J. M. S. Nogueira, "Vehicular networks using the IEEE 802.11p standard: an experimental analysis", Veh. Commun., vol. 1, no. 2, pp. 91-96, 2014 (doi: 10.1016/j.vehcom.2014.04.001).

[14] M. E. Renda, G. Resta, P. Santi, F. Martelli, and A. Franchini, "IEEE 802.11p VANets: experimental evaluation of packet interreception time", Comp. Commun., vol. 75, no. 1, pp. 26-38, 2016 (doi: 10.1016/j.comcom.2015.06.003).

[15] T. T. Almeida, L. C. Gomes, F. M. Ortiz, J. G. R. Júnior, and L. H M. K. Costa, "IEEE 802.11p performance evaluation: simulations vs. real experiments", in Proc. 21st IEEE Int. Conf. Intelli. Transport. Syst. ITSC 2018, Maui, Hawaii, USA, 2018, pp. 3840-3845 (doi: 10.1109/ITSC.2018.8569676).

[16] D. Wang, Z. Li, and Y. Chen, "Design and implementation of a location based service business management platform", in Proc. 4th Int. Conf. on Syst. \& Informat. ICSAI 2017, Hangzhou, China, 2017, pp. 1631-1635 (doi: 10.1109/ICSAI.2017.8248545).

[17] C.-M. Huang, D.-T. Dao, and C.-M. Mai, "Location-based service (LBS) data sharing using the k-member-limited clustering mechanism over the 4G and WiFi hybrid wireless mobile network", in Proc. Int. Conf. Inform. Netw. ICOIN 2017, Da Nang, Vietnam, 2017 (doi: 10.1109/ICOIN.2017.7899550).

[18] S. Wang et al., "N-in-one: a novel location-based-service", IEEE Trans. on Veh. Technol., vol. 67, no. 6, pp. 5274-5286, 2018 (doi: 10.1109/TVT.2017.2737017).

[19] S. Ishida et al., "Implementation of on-demand indoor locationbased service using ad-hoc wireless positioning network", in Proc. 11th Int. Conf. Ubiquitous Intell. \& Comput. (UIC 2014) and 11th Int. Conf. on Autonom. and Trust. Comput. and 14th Int. Conf. on Scal. Comput. and Commun. and its Assoc. Worksh., Bali, Indonesia, 2014, pp. 34-41 (doi: 10.1109/UIC-ATC-ScalCom.2014.96).

[20] A. Mena et al., "Interactive geo-location based service application as pervasive computing through mobile devices", in Proc. Chilean Conf. on Elec., Electron. Engin., Inform. \& Commun. Technol. CHILECON 2017, Pucon, Chile, 2017 (doi: 10.1109/CHILECON.2017.8229529).

[21] Y. Kaneko, K. Harumoto, S. Fukumura, S. Shimojo, and S. Nishio, "A location-based peer-to-peer network for context-aware services in a ubiquitous environment", in Proc. Symp. Appl. \& Internet Worksh. SAINT-W 2005, Trento, Italy, 2005 (doi: 10.1109/SAINTW.2005.1620013).

[22] A. Kovačević, N. Liebau, and R. Steinmetz, "Globase.KOM - a P2P overlay for fully retrievable location-based search", in Proc. 7th IEEE Int. Conf. on Peer-to-Peer Comput. P2P 2007, Galway, Ireland, 2007, pp. 87-94 (doi: 10.1109/P2P.2007.18). 
[23] C.-S. Wang, W.-D. Chen, and C.-L. Chen, "Location-based P2P mobile navigation system", in Proc. Int. Symp. Compu. Sci. \& Soc. ISCCS 2011, Kota Kinabalu, Malaysia, 2011 (doi: 10.1109/ISCCS.2011.104).

[24] C.-S. Wang, C.-L. Chen, and D.-J. Deng, "P2P-based mobile navigation system with location service", Peer-to-Peer Network. and Appl., vol. 8, no. 1, pp. 22-31, 2015 (doi: 10.1007/s12083-013-0204-8).

[25] J. Rosenberg, R. Mahy, P. Matthews, and D. Wing, "Session traversal utilities for NAT (STUN)”, RFC 5389, IETF, Oct. 2008.

[26] R. Mahy, P. Matthews, and J. Rosenberg, "Traversal using relays around NAT (TURN): relay extensions to session traversal utilities for NAT (STUN)", RFC 5766, IETF, Apr. 2010.

[27] Fujitsu Cloud Technologies, Ltd., NIFCLOUD, homepage [Online]. Available: https://cloud.nifty.com

[28] NTT Communications Corp., Enterprise Cloud SkyWay, homepage [Online]. Available: https://webrtc.ecl.ntt.com

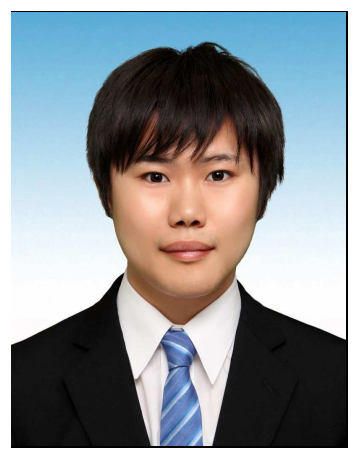

Yusuke Shimomura received his B.E. degree in Electronic Information Systems from the Shibaura Institute of Technology, Tokyo, Japan, in 2017. $\mathrm{He}$ is presently a master's course student at the Graduate School of Engineering and Science, Shibaura Institute of Technology, Tokyo, Japan. His research interests are in peerto-peer networks and their applications.
E-mail: mf17037@shibaura-it.ac.jp

Graduate School of Engineering and Science

Shibaura Institute of Technology

Saitama, Japan

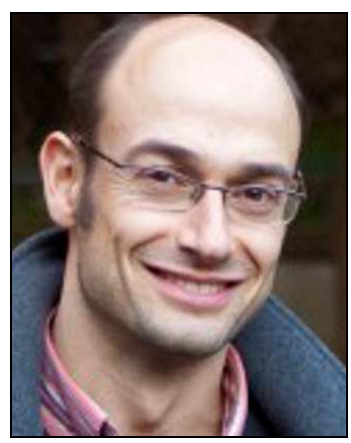

Olivier Fourmaux has been an Associate Professor at Sorbonne Université, France, since 2003. Before, he was an Assistant Professor at Institut Galilee, Université Paris 13, France. He received his Ph.D. degree in Computer Networking in 1998 and his M.Sc. degree in computer systems in 1995, both from UPMC. His research interests cover content delivery networks, $\mathrm{P} 2 \mathrm{P}$ networks, active networks and multimedia in high-speed networks. He is a member of the Network and Performance group of the LIP6 Laboratory (CNRS-Sorbonne Université)

E-mail: olivier.fourmaux@ @orbonne-universite.fr Laboratoire d'Informatique de Paris 6

Sorbonne Université

Paris, France

Takumi Miyoshi - for biography, see this issue, p. 22. 